\title{
Trend and Predictors of the Utilization of Endoscopic Retrograde Cholangiopancreatography in Acute Pancreatitis Hospitalizations
}

Ashish Sharma ${ }^{1}$, Jigisha H. Rakholiya ${ }^{2}$, Apoorva Madapu ${ }^{3}$, Shivy Sharma ${ }^{4}$, Anil Jha 5

1. Internal Medicine, Yuma Regional Medical Center, Yuma, USA 2. Internal Medicine, Mayo Clinic, Rochester, USA 3. Internal Medicine, Huntsville Hospital, Huntsville, USA 4. Internal Medicine, Marshfield Clinic Health System, Marshfield, USA 5. Internal Medicine, Lawrence General Hospital, Lawrence, USA

Corresponding author: Ashish Sharma, ashishsharma1412@gmail.com

\section{Abstract \\ Background}

Acute pancreatitis is a sudden inflammation of the pancreas, and biliary pancreatitis remains the most common cause of acute pancreatitis. Endoscopic retrograde cholangiopancreatography (ERCP) is both a diagnostic and therapeutic invasive procedure to evaluate and treat pancreaticobiliary system diseases. ERCP is very commonly used in acute pancreatitis with coexisting acute cholangitis or biliary obstruction. There was a need for a nationwide study to evaluate ERCP utilization trends and health-care costs among acute pancreatitis patients.

\section{Aim}

We sought to determine the prevalence trend, hospitalization cost and stay, and predictors of utilization of ERCP amongst patients with acute pancreatitis.

\section{Methods}

We performed a population-based retrospective analysis of national data in adult acute pancreatitis hospitalizations. We evaluated the characteristics of the ERCP cohort, prevalence trend, and hospital utilization cost and stay using univariate analysis. Multivariable survey logistic regression analysis was performed to evaluate predictors of utilization for ERCP among acute pancreatitis hospitalization.

\section{Results}

Among 2,632,309 hospitalizations for acute pancreatitis, 49108 (1.87\%) had ERCP. The prevalence trend of ERCP declined from $3.88 \%$ in 2003 to $0.97 \%$ in 2014. $\left(\mathrm{p}_{\text {Trend }}<0.0001\right)$. Patients with ERCP were older ( $>55$ -

Review began 09/18/2020 Review ended 11/01/2020 Published 11/10/2020

\section{(c) Copyright 2020}

Sharma et al. This is an open access article distributed under the terms of the Creative Commons Attribution License CC-BY 4.0., which permits unrestricted use, distribution, and reproduction in any medium, provided the original author and source are credited. years old) (53.01\% vs 39.36\%; $<0.0001)$, female (58.45\% vs $48.04 \%$; p<0.0001), Hispanic (16.30\% vs $12.86 \%$; $\mathrm{p}<0.0001$ ), utilizing Medicare (40.29\% vs 31.88\%; $\mathrm{p}<0.0001$ ), elective admission (8.15\% vs 4.98\%; $\mathrm{p}<0.0001)$, and with gallbladder etiology (65.98\% vs 26.06\%; $\mathrm{p}<0.0001)$. Acute pancreatitis hospitalization with ERCP had a higher cost of utilization (Cost diff: $+\$ 25077 ; \mathrm{p}<0.0001)$ and mean stay $\left(\operatorname{LOS}_{\text {diff: }}{ }^{+3.5}\right.$ days; $\left.<<0.0001\right)$. In regression analysis, old adults [Odds ratio(OR):1.087; Confidence interval (CI):1.008-1.173), Hispanic (OR:1.086; CI:1.019-1.156), asian (OR:1.146; CI:1.007-1.304), female (OR:1.074; CI:1.028-1.122), elective admission (OR:1.649; CI:1.524-1.785), gallbladder etiology (OR:4.437; CI:4.224-4.662), concurrent chronic pancreatitis (OR:1.643; CI:1.536-1.757), systemic inflammatory response syndrome (SIRS) (OR:1.264; CI:1.112-1.436), pleural effusion (OR:1.874; CI:1.231-2.854), and portal vein thrombosis (OR:1.646; CI:1.221-2.219).

\section{Conclusion}

In nationwide data, we have found a decreased utilization trend and higher hospital utilization cost and stay associated with ERCP. The predictors of utilization will be helpful to examine the cost-utility of ERCP, especially with the advent of acute pancreatitis treatment systems to mitigate the health care burden.

Categories: Internal Medicine, Gastroenterology, Epidemiology/Public Health

Keywords: acute pancreatitis, endoscopic retrograde cholangiopancreatography, gallstone, nationwide inpatient sample (nis), observational study

\section{Introduction}

Gastrointestinal (GI) diseases account for significant health care utilization and spending [1]. In 2015, health care expenditures for GI conditions summed $\$ 135.9$ billion, and among the 22 condition categories 
available, biliary tract disease was one of the top-five most expensive categories, costing about $(\$ 10.3$ billion) [1]. Among the gastrointestinal diseases, acute pancreatitis is one of the most common causes of inpatient hospital admissions in the United States [2-3]. Acute pancreatitis was alone responsible for 279,000 hospital admissions, $0.7 \%$ in-hospital mortality, U.S. $\$ 2.64$ billion in aggregate charges, and a three-day median hospital length of stay (LOS) in 2014 [1].

Acute pancreatitis treatment ranges from conservative medical management to more invasive treatment [34]. Endoscopic retrograde cholangiopancreatography (ERCP) is one of the commonly performed invasive procedures for the diagnosis and treatment of biliary and pancreatic diseases [2,4]. Both the therapeutic and diagnostic intervention capabilities of ERCP gives it an edge over other diagnostic utilities, however, ERCP utilization has been observed to be affected by the newly emerging diagnostic studies, such as magnetic resonance cholangiopancreatography (MRCP) and endoscopic ultrasonography (EUS), along with other patient and non-patient related factors [1-2].

The primary aim of our study is to investigate the yearly utilization trend and predictors of utilization of ERCP among patients with acute pancreatitis, and the secondary outcome is to evaluate the outcomes of the hospitalizations.

\section{Materials And Methods}

Nationwide Inpatient Sample (NIS) data between January 2003 and December 2014 were obtained from the Agency for Healthcare Research and Quality's Healthcare Cost and Utilization Project. It is the publicly available all-payer inpatient care database in the US and contains discharge-level data provided by states participating in the Healthcare Cost and Utilization Project. This administrative dataset contains data on approximately 8-million hospitalizations in 1,000 hospitals chosen to approximate a $20 \%$ stratified sample of all US community hospitals, representing more than $95 \%$ of the national population. Detailed information on NIS is available at http://www.hcup-us.ahrq.gov/db/nation/nis/nisdde.jsp.

\section{Study population}

We used the 9th revision of the International Classification of Diseases, clinical modification (ICD-9$\mathrm{CM}$ ) code to identify adult patients admitted to the hospital with a primary diagnosis of acute pancreatitis (ICD-9-CM code 577.0). Similarly, patients who utilized ERCP were identified using ICD-9 procedure codes 51.10. We have considered acute pancreatitis as a primary diagnosis for which hospitalization occurred and have seen ERCP amongst those patients. This population does not cover post-ERCP pancreatitis or ERCP in other urgent conditions. We used ICD-9-CM codes to identify independent predictors (covariates), including the comorbidities of hypertension, diabetes mellitus, hypercholesterolemia/dyslipidemia, smoker (current/past), drug abuse, acquired immunodeficiency syndrome, alcohol abuse/dependence and withdrawal, ischemic heart diseases, and chronic pancreatitis. Similarly, we identified complications like hypercalcemia, acute renal failure, shock, systemic inflammatory response syndrome, ascites, pleural effusion, respiratory distress/failure, and portal vein thrombosis. Table 1 mentions ICD-9-CM codes for all the concurrent conditions - adults $<18$ years and admissions with missing data for age, gender, and race were excluded. The sample size was based on the available data. 


\section{Cureus}

Acute pancreatitis

Gallstone

Hypertension

Diabetes

Obesity

Hypercholesterolemia

Drug Abuse

Alcohol Abuse

Current or Past Smoker

Acquired Immunodeficiency Syndrome (AIDS)

Ischemic Heart Disease

End-Stage Renal Disease

Chronic Kidney Disease

Alcohol Withdrawal

Chronic Pancreatitis

Complications (\%)

Hypercalcemia

Acute Renal Failure

Shock

Systemic Inflammatory Response Syndrome

Ascites

Pleural Effusion

Respiratory Distress and Respiratory Failure

Portal Vein Thrombosis
577.0

574

401-405

249-250

278.0

272.0,272.1,272.2

304.X, 305.2-305.9

V11.3, 303.X, 305.X

V15.82, 305.1

042, V08

410-414

585.6

$585.1-5,585.9$

291.81

577.1

275.42

584.5-9

785.5

995.9

789.5

511.8

518.8

452

\section{TABLE 1: ICD-9-CM codes used in this analysis}

ICD-9-CM: International Classification of Diseases, clinical modification 9

\section{Patient and hospital characteristics}

Patient characteristics of interest were age, sex, race, insurance status, and concomitant diagnoses, as defined above. The race was defined by White (referent), African American, Hispanic, Asian or Pacific Islander, and Native American. Insurance status was defined by Medicare (referent), Medicaid, Private Insurance, and Other/Self-pay/No charge. We defined the severity of comorbid conditions using Deyo's modification of the Charlson's comorbidity index (CCI) (Table 2). Healthcare Cost and Utilization Project NIS contains data on total charges for each hospital in the databases, representing the amount that hospitals billed for services. 


\section{Cureus}

\begin{tabular}{|c|c|c|}
\hline Condition & ICD-9-CM Codes & Charlson Score \\
\hline Myocardial Infarction & $410-410.9$ & 1 \\
\hline Congestive Heart Failure & $428-428.9$ & 1 \\
\hline Peripheral Vascular Disease & 433.9, $441-441.9,785.4$, V43.4 & 1 \\
\hline Cerebrovascular Disease & $430-438$ & 1 \\
\hline Dementia & $290-290.9$ & 1 \\
\hline Chronic Pulmonary Disease & $490-496,500-505,506.4$ & 1 \\
\hline Rheumatologic Disease & $710.0,710.1,710.4,714.0-714.2,714.81,725$ & 1 \\
\hline Peptic Ulcer Disease & $531-534.9$ & 1 \\
\hline Mild Liver Disease & $571.2,571.5,571.6,571.4-571.49$ & 1 \\
\hline Diabetes & $250-250.3,250.7$ & 1 \\
\hline Diabetes With Chronic Complications & $250.4-250.6$ & 2 \\
\hline Hemiplegia or Paraplegia & $344.1,342-342.9$ & 2 \\
\hline Chronic Kidney Disease & $582-582.9,583-583.7,585,586,588-588.9$ & 2 \\
\hline Any Malignancy Including Leukemia and Lymphoma & $140-172.9,174-195.8,200-208.9$ & 2 \\
\hline Moderate or Severe Liver Disease & $572.2-572.8$ & 3 \\
\hline Metastatic Solid Tumor & 196-199.1 & 6 \\
\hline Acquired Immunodeficiency Syndrome & $042-044.9$ & 6 \\
\hline
\end{tabular}

TABLE 2: Deyo's modification of Charlson's comorbidity index $(\mathrm{CCl})$

\section{Outcomes}

This study's primary aim was to evaluate the characteristics of patients with ERCP in acute pancreatitis patients, yearly utilization trends, predictors of ERCP utilization, and prevalent complications amongst acute pancreatitis underwent ERCP. The secondary aim was to evaluate the outcomes like mortality, morbidity, disability (loss of function), discharge disposition, length of stay (LOS), and cost of hospitalization associated with ERCP (years 2003-2014). The comparison of disability/loss of function was investigated by All Patient Refined Diagnosis Related Groups (APR-DRGs) severity. APR-DRGs were assigned using software developed by 3M Health Information Systems (Wallingford, Connecticut), where score 1 indicates minor loss of function, 2 - moderate, 3 - major, and 4 - extreme loss of function. Morbidity is defined as the length of stay $>10$ days ( $>90$ percentile) and discharges other than home (short-term hospital, skilled nursing facility, intermediate care facility). Discharge disposition was defined by discharge to home vs. non-home [5].

\section{Statistical analysis}

All statistical analyses were performed using the weighted survey methods in SAS (version 9.4; SAS Institute, Cary, North Carolina). The p-values of $<0.05$ were considered significant. Univariate analysis of differences between categorical variables was tested using the chi-square test. Analysis of differences between continuous variables (age, LOS, and cost of hospitalization) was tested using the paired student's ttest. Mixed-effects survey logistic regression models with weighted analysis were used to evaluate ERCP utilization predictors amongst acute pancreatitis hospitalizations during 2003-2014. We included demographics (age, gender, race), patient-level hospitalization variables (admission day, primary payer, admission type, median household income category), hospital-level variables (hospital region, teaching versus non-teaching hospital, hospital bed size), comorbidities, concurrent conditions, complications, and CCI. The goodness of fit of the model was evaluated by the c-value.

\section{Results}

\section{Disease hospitalizations}

We found a total of 2,632,309 hospitalizations (unweighted:535,136) due to acute pancreatitis from 2003 to 


\section{Cureus}

2014 after excluding patients aged $<18$ years and admissions with missing data for age, gender, and ethnicity. Out of 2,632,309 acute pancreatitis hospitalizations, the prevalence of ERCP utilization was 49,107 (1.87\%) (Figure 1).

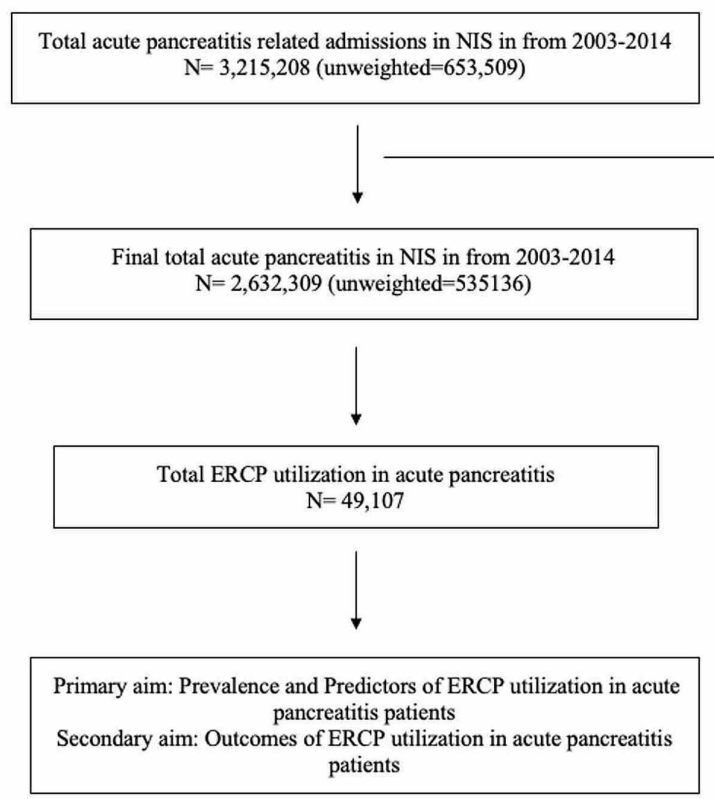

FIGURE 1: Flowchart showing details of the final study population extracted from NIS with inclusion and exclusion criteria for the study NIS: Nationwide Inpatient Sample

\section{Prevalence trend}

We analyzed the trend of utilization of ERCP in acute pancreatitis hospitalizations. As shown in Figure 2, the trend of utilization of ERCP is decreasing from 2003 to 2014 (utilization of ERCP, 3.88\% in 2003 to $0.97 \%$ in 2014; p-trend $<0.0001$ ).

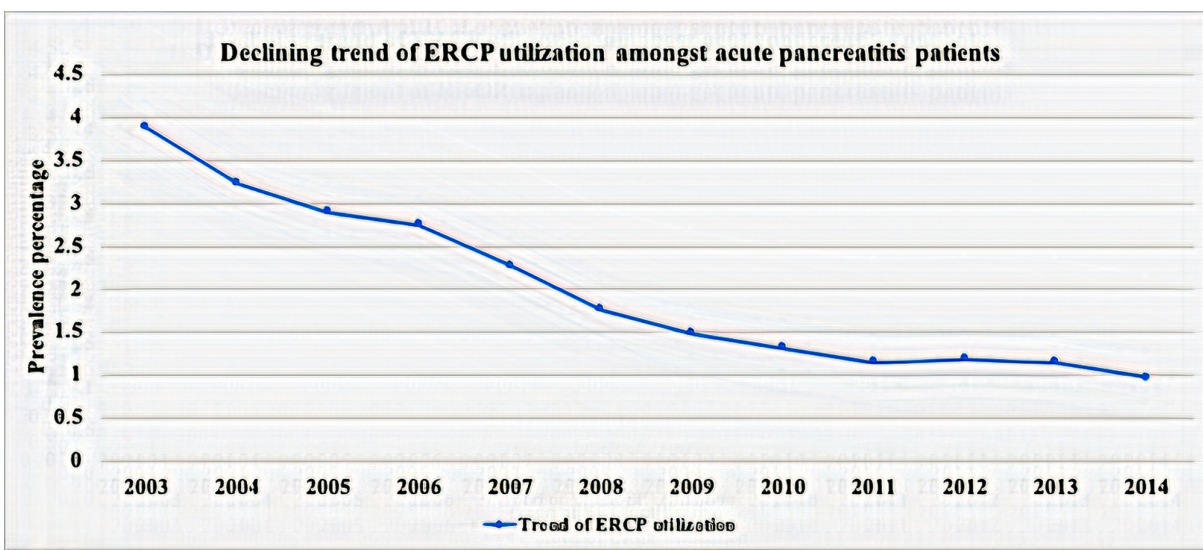

FIGURE 2: Utilization trend of ERCP amongst hospitalized patients with acute pancreatitis

ERCP: endoscopic retrograde cholangiopancreatography

Demographics, patient and hospital characteristics, and comorbidities

The utilization of ERCP was more common in older age groups (mean age 57 vs 52 ; $\mathrm{p}<0.0001$ ). ERCP utilization was more in patients in the age group > 55 years than the age group $18-55$ years $(2.50 \%$ vs $1.45 \%$; $\mathrm{p}<0.0001)$. Amongst the patients with acute pancreatitis who underwent ERCP, females were more likely 


\section{Cureus}

than males (2.26\% vs 1.50\%; p 0.0001) and Asian and Pacific Islanders as compared to white, African American, Hispanic and native American (2.60\% vs $1.88 \%$ vs $1.31 \%$ vs $2.33 \%$ vs $1.29 \%$; $p<0.0001$ ), had Medicare (2.35\%; $\mathrm{p}<0.0001)$, had elective admissions as compared to emergency admissions (3.01\% vs $1.80 \%$; $\mathrm{p}<0.0001)$, admissions on weekdays as compared to weekends ( $1.90 \%$ vs $1.78 \%$; $<<0.0001)$, and in urbanteaching hospitals as compared to rural and urban non-teaching ( $2.20 \%$ vs $0.74 \%$ vs $1.94 \%$; $<0.0001)$. The prevalence of ERCP utilization is higher than those with non-ERCP utilization in females (58.45\% vs 48.04\%; $\mathrm{p}<0.0001)$, Hispanic (16.30\% vs $12.86 \%$; $\mathrm{p}<0.0001$ ), Medicare (40.29\% vs $31.88 \%$; $\mathrm{p}<0.0001$ ), elective admissions (8.15\% vs $4.98 \%$; $\mathrm{p}<0.0001$ ), weekdays (75.17\% vs $24.83 \%$; $\mathrm{p}<0.0001$ ), and urban-teaching hospital location (47.21\% vs 39.94\%; $\mathrm{p}<0.0001)$.

Comorbidities like gallstone and gallbladder diseases (65.98\% vs $26.06 \%$; $p<0.0001)$ and ischemic heart disease (14.41\% vs $12.10 \%$; $\mathrm{p}<0.0001$ ) were having a higher utilization of ERCP than those with non-ERCP utilization. Comorbidities like hypertension (49.25\% vs $50.45 \%$; $\mathrm{p}<0.0001$ ), diabetes $(22.36 \%$ vs $25.93 \%$; $\mathrm{p}<0.0001$ ), hypercholesterolemia (7.52\% vs $10.22 \%$; $\mathrm{p}<0.0001)$, drug abuse ( $2.47 \%$ vs $6.06 \%$; $<<0.0001)$, alcohol abuse (9.72\% vs $28.26 \%$; $\mathrm{p}<0.0001)$, current or past smoker $(19.25 \%$ vs $31.02 \%$; $<<0.0001)$, acquired immunodeficiency syndrome $(0.46 \%$ vs $0.67 \%$; $p<0.0001)$, end stage renal disease $(1.23 \%$ vs $1.65 \%$; $\mathrm{p}<0.0001)$, chronic kidney disease (3.46\% vs 3.95\%; $\mathrm{p}<0.0001)$, alcohol withdrawal $(0.49 \%$ vs $3.72 \%$; $\mathrm{p}<0.0001)$, and chronic pancreatitis $(11.77 \%$ vs $13.43 \%$; $\mathrm{p}<0.0001)$ were having a lower utilization of ERCP than those with non-ERCP utilization. Complications like shock (1.03\% vs 0.71\%; $<<0.0001)$, systemic inflammatory response syndrome (3.60\% vs $2.51 \%$; $<<0.0001)$, ascites $(3.07 \%$ vs $2.57 \%$; $<<0.0001)$, pleural effusion ( $0.29 \%$ vs $0.11 ; \mathrm{p}<0.0001)$, respiratory distress and failure ( $3.33 \%$ vs $2.94 \%$; $\mathrm{p}<0.0001)$, and portal vein thrombosis $(0.50 \%$ vs $0.30 \%$; $<<0.0001)$ were higher among ERCP utilization than those with non-ERCP utilization (Table 3).

\begin{tabular}{|c|c|c|c|c|}
\hline & ERCP & Non-ERCP & Total & p-value \\
\hline Acute Pancreatitis Weighted (\%) & 49107 (1.87) & 2583202 (98.13) & $2632309(100)$ & $<0.0001$ \\
\hline \multicolumn{5}{|l|}{ Demographics of Patients } \\
\hline Age Mean (SD) (Years) & $57+/-18$ & $52+/-17$ & & $<0.0001$ \\
\hline Age Group (Years) & & & & $<0.0001$ \\
\hline 18-55 years & 23076 (46.99) & $1566559(60.64)$ & 1589635 (60.39) & \\
\hline$>55$ Years & 26032 (53.01) & 1016642 (39.36) & 1042674 (39.61) & \\
\hline Gender (\%) & & & & $<0.0001$ \\
\hline Male & 20403 (41.55) & 1342264 (51.96) & 1362667 (51.77) & \\
\hline Female & 28704 (58.45) & 1240808 (48.04) & 1269512 (48.23) & \\
\hline Race (\%) & & & & $<0.0001$ \\
\hline White & 32294 (68.19) & 1681834 (66.99) & 1714128 (67.01) & \\
\hline African American & $5792(12.23)$ & $436980(17.41)$ & $442772(17.31)$ & \\
\hline Hispanic & 7718 (16.30) & 322971 (12.86) & $330689(12.93)$ & \\
\hline Asian or Pacific Islander & $1286(2.72)$ & 48193 (1.92) & 49479 (1.93) & \\
\hline Native American & $269(0.57)$ & $20548(0.82)$ & $20817(0.81)$ & \\
\hline \multicolumn{5}{|l|}{ Characteristics of Patients } \\
\hline Median Household Income Category for Patient's Zip Code (\%) & & & & $<0.0001$ \\
\hline 0 -25th percentile & $13871(28.88)$ & $801293(31.83)$ & $815164(31.77)$ & \\
\hline 26-50th percentile & $12130(25.25)$ & $659970(26.21)$ & $672100(26.20)$ & \\
\hline 51-75th percentile & 11826 (24.62) & 578597 (22.98) & $590423(23.01)$ & \\
\hline 76-100th percentile & $10205(21.25)$ & $477773(18.98)$ & $487978(19.02)$ & \\
\hline Primary Payer (\%) & & & & $<0.0001$ \\
\hline Medicare & 19750 (40.29) & $821389(31.88)$ & 841139 (32.04) & \\
\hline Medicaid & $6632(13.53)$ & 420525 (16.32) & $427157(16.27)$ & \\
\hline
\end{tabular}




\section{Cureus}

\begin{tabular}{|c|c|c|c|c|}
\hline Private Insurance & 17265 (35.22) & 859345 (33.35) & 876610 (33.39) & \\
\hline Other/Self-pay/No Charge & $5378(10.97)$ & 475215 (18.44) & 480593 (18.30) & \\
\hline Admission type (\%) & & & & $<0.0001$ \\
\hline Non-Elective & 44991 (91.85) & 2449856 (95.02) & 2494847 (94.96) & \\
\hline Elective & $3990(8.15)$ & $128454(4.98)$ & 132444 (5.04) & \\
\hline Admission Day (\%) & & & & $<0.0001$ \\
\hline Weekday & 36914 (75.17) & 1910348 (73.95) & 1947262 (73.98) & \\
\hline Weekend & $12193(24.83)$ & 672854 (26.05) & 685047 (26.02) & \\
\hline \multicolumn{5}{|l|}{ Characteristics of Hospitals } \\
\hline Bed Size of the Hospital (\%) * & & & & $<0.0001$ \\
\hline Small & $4445(9.07)$ & 401046 (15.59) & 405491(15.47) & \\
\hline Medium & $13369(27.27)$ & $715471(27.82)$ & $728840(27.81)$ & \\
\hline Large & $31209(63.66)$ & 1455584 (56.59) & 1486793 (56.72) & \\
\hline Hospital Location \& Teaching Status (\%) & & & & $<0.0001$ \\
\hline Rural & $2844(5.80)$ & 379024 (14.74) & 381868 (14.57) & \\
\hline Urban Non-Teaching & 23035 (46.99) & $1165889(45.33)$ & 1188924 (45.36) & \\
\hline Urban Teaching & $23144(47.21)$ & 1027187 (39.94) & $1050331(40.07)$ & \\
\hline Hospital Region (\%) & & & & $<0.0001$ \\
\hline Northeast & $11498(23.41)$ & 513005 (19.86) & $524503(19.93)$ & \\
\hline Midwest & $7164(14.59)$ & $443519(17.17)$ & 450683 (17.12) & \\
\hline South & 20093 (40.92) & 1090298 (42.21) & 1110391 (42.18) & \\
\hline West & $10353(21.08)$ & $536379(20.76)$ & $546732(20.77)$ & \\
\hline \multicolumn{5}{|l|}{ Comorbidities of Patients (\%) } \\
\hline Hypertension & $24184(49.25)$ & 1303135 (50.45) & 1327319 (50.42) & $<0.0001$ \\
\hline Diabetes & $10982(22.36)$ & 669914 (25.93) & $680896(25.87)$ & $<0.0001$ \\
\hline Obesity & $5234(10.66)$ & 279294 (10.81) & $284528(10.81)$ & 0.2778 \\
\hline Hypercholesterolemia & 3691 (7.52) & 264066 (10.22) & 267757 (10.17) & $<0.0001$ \\
\hline Drug Abuse & $1215(2.47)$ & $156616(6.06)$ & $157831(6.00)$ & $<0.0001$ \\
\hline Alcohol Abuse & 4775 (9.72) & 730103 (28.26) & 734878 (27.92) & $<0.0001$ \\
\hline Current or Past Smoker & 9455 (19.25) & 801391 (31.02) & $810846(30.80)$ & $<0.0001$ \\
\hline Gallstone & $32400(65.98)$ & $673263(26.06)$ & $705663(26.81)$ & $<0.0001$ \\
\hline Acquired Immunodeficiency Syndrome & $223(0.46)$ & $17389(0.67)$ & $17612(0.67)$ & $<0.0001$ \\
\hline Ischemic Heart Disease & 7075 (14.41) & 312548 (12.10) & 319623 (12.14) & $<0.0001$ \\
\hline End-Stage Renal Disease & $604(1.23)$ & $42515(1.65)$ & 43119 (1.64) & $<0.0001$ \\
\hline Chronic Kidney Disease & $1698(3.46)$ & $102163(3.95)$ & 103861 (3.95) & $<0.0001$ \\
\hline Alcohol Withdrawal & $241(0.49)$ & 96142 (3.72) & $96383(3.66)$ & $<0.0001$ \\
\hline Chronic Pancreatitis & $5780(11.77)$ & 346851 (13.43) & 352631 (13.40) & $<0.0001$ \\
\hline \multicolumn{5}{|l|}{ Complications (\%) } \\
\hline Hypercalcemia & $176(0.36)$ & $15825(0.61)$ & $16001(0.61)$ & $<0.0001$ \\
\hline Acute Renal Disease & $3407(6.94)$ & $183301(7.10)$ & $186708(7.09)$ & 0.1763 \\
\hline
\end{tabular}




\section{Cureus}

$\begin{array}{lllll}\text { Shock } & 504(1.03) & 18211(0.71) & 18715(0.71) & <0.0001 \\ \text { Systemic Inflammatory Response Syndrome } & 1769(3.60) & 64887(2.51) & 66656(2.53) & <0.0001 \\ \text { Ascites } & 1506(3.07) & 66273(2.57) & 67779(2.57) & <0.0001 \\ \text { Pleural Effusion } & 143(0.29) & 2868(0.11) & 3011(0.11) & <0.0001 \\ \text { Respiratory Distress/Failure } & 1637(3.33) & 76012(2.94) & 77649(2.95) & <0.0001 \\ \text { Portal Vein Thrombosis } & 245(0.50) & 7791(0.30) & 8036(0.31) & <0.0001 \\ \text { Deyo's Charlson Comorbidity Index (CCl) } & & & & <0.0001 \\ 0 & 27293(55.58) & 1373428(53.17) & 1400721(53.21) & \\ 1 & 12326(25.10) & 703107(27.22) & 715433(27.18) & \\ 2 & 4755(9.68) & 255546(9.89) & 260301(9.89) & \\ 3 & 2168(4.41) & 112035(4.34) & 114203(4.34) & \\ 4 & 1282(2.61) & 62336(2.41) & 63618(2.42)\end{array}$

TABLE 3: Characteristics of patients with endoscopic retrograde cholangiopancreatography (ERCP) in acute pancreatitis hospitalizations

* Bed size of the hospital indicates the number of hospital beds, which varies depending on hospital location (Rural/Urban), teaching status (Teaching/Non-Teaching), and Region (Northeast/Midwest/Southern/Western) The percentage in bracket is column \% indicates the direct comparison between ERCP vs. Non-ERCP amongst acute pancreatitis patients.

\section{Regression model derivation for predictors of ERCP utilization}

In multivariate regression analysis, old adults (aOR:1.09; 95\%CI:1.01-1.17; $\mathrm{p}=0.0306)$, male (aOR:1.07; 95\%CI:1.03-1.12; $\mathrm{p}=0.0015$ ), Hispanic (aOR:1.09; 95\%CI:1.02-1.16; $\mathrm{p}=0.0104)$, Asian or Pacific Islander (aOR:1.15; 95\%CI:1.01-1.30; $\mathrm{p}=0.0391$ ), medium bed size of hospital (aOR:1.61; 95\%CI:1.49-1.74; $\mathrm{p}<0.0001$ ), large bed size of hospital (aOR:1.86; 95\%CI:1.73-2.00; p<0.0001), urban non-teaching hospital (aOR:2.67; 95\%CI:2.43-2.94; $\mathrm{p}<0.0001$ ), urban teaching hospital (aOR:2.98; 95\%CI:2.71-3.28; $\mathrm{p}<0.0001$ ), gallstone and gallbladder diseases (aOR:4.44; 95\%CI:4.22-4.66; $\mathrm{p}<0.0001$ ), and chronic pancreatitis (aOR:1.64; 95\%CI:1.54-1.76; $\mathrm{p}<0.0001$ ) have higher odds of utilization of ERCP.

We have noticed a lower chance of ERCP utilization amongst African Americans (aOR:0.87; 95\%CI:0.810.93; p<0.0001), other/self-pay/no charge (aOR:0.76; 95\%CI:0.69-0.82; $<<0.0001)$, weekend (aOR: 0.94; 95\%CI:0.90-0.99; $\mathrm{p}=0.0134$ ), hypertension (aOR:0.93; 95\%CI:0.89-0.98; $\mathrm{p}=0.0042$ ), diabetes (aOR:0.88; 95\%CI:0.83-0.93; $\mathrm{p}<0.0001$ ), obesity (aOR:0.81; 95\%CI:0.76-0.87; $<<0.0001$ ), hypercholesterolemia (aOR:0.80; 95\%CI:0.74-0.87; $\mathrm{p}<0.0001$ ), drug abuse (aOR:0.81; 95\%CI:0.70-0.92; $\mathrm{p}=0.0016$ ), alcohol abuse (aOR:0.54; 95\%CI:0.50-0.58; $<<0.0001$ ), current or past smoker (aOR:0.80; 95\%CI:0.76-0.84; $<<0.0001$ ), end stage renal disease (aOR:0.70; 95\%CI:0.58-0.85; $\mathrm{p}=0.0004)$, chronic kidney disease (aOR:0.73; 95\%CI:0.650.83; $\mathrm{p}<0.0001$ ), and alcohol withdrawal (aOR:0.38; 95\%CI:0.28-0.52; $\mathrm{p}<0.0001)$ than non-ERCP utilization.

ERCP utilization was linked with having higher odds of complications like SIRS (aOR:1.26; 95\%CI:1.11-1.44; $\mathrm{p}=0.0003$ ), pleural effusion (aOR:1.87; 95\%CI:1.23-2.85; $\mathrm{p}=0.0034$ ) and portal vein thrombosis (aOR:1.65; 95\%CI:1.22-2.22; $\mathrm{p}=0.0011$ ). The $\mathrm{c}$-statistic, which is used to validate the accuracy of the regressions, was 0.77 (>0.5), which indicates a good model (Table 4).

\begin{tabular}{|c|c|c|c|c|}
\hline & \multirow[t]{2}{*}{ Odds Ratio } & \multicolumn{2}{|c|}{ Confidence Interval 95\% } & \multirow[t]{2}{*}{ p-value } \\
\hline & & LL & UL & \\
\hline Age (Years) & 1.00 & 1.00 & 1.01 & 0.0017 \\
\hline 18-55 years & \multicolumn{4}{|l|}{ Reference } \\
\hline$>55$ years & 1.09 & 1.01 & 1.17 & 0.0306 \\
\hline Gender & & & & \\
\hline
\end{tabular}




\section{Cureus}

\begin{tabular}{|c|c|c|c|c|}
\hline \multirow{2}{*}{$\begin{array}{l}\text { Female } \\
\text { Male }\end{array}$} & \multicolumn{4}{|c|}{ Reference } \\
\hline & 1.07 & 1.03 & 1.12 & 0.0015 \\
\hline \multicolumn{5}{|l|}{ Race } \\
\hline White & \multicolumn{4}{|c|}{ Reference } \\
\hline African American & 0.87 & 0.81 & 0.93 & $<0.0001$ \\
\hline Hispanic & 1.09 & 1.02 & 1.16 & 0.0104 \\
\hline Asian or Pacific Islander & 1.15 & 1.01 & 1.30 & 0.0391 \\
\hline Native American & 1.01 & 0.76 & 1.34 & 0.9394 \\
\hline \multicolumn{5}{|c|}{ Median Household Income Category for Patient's Zipcode } \\
\hline 0 -25th percentile & \multicolumn{4}{|c|}{ Reference } \\
\hline 26-50th percentile & 0.98 & 0.93 & 1.04 & 0.5778 \\
\hline 51-75th percentile & 0.97 & 0.91 & 1.03 & 0.3402 \\
\hline 76-100th percentile & 0.88 & 0.83 & 0.94 & 0.0002 \\
\hline \multicolumn{5}{|l|}{ Primary Payer } \\
\hline Medicare & \multicolumn{4}{|c|}{ Reference } \\
\hline Medicaid & 0.97 & 0.89 & 1.05 & 0.4185 \\
\hline Private Insurance & 1.03 & 0.97 & 1.10 & 0.2828 \\
\hline Other/Self-pay/No charge & 0.76 & 0.69 & 0.82 & $<0.0001$ \\
\hline \multicolumn{5}{|l|}{ Admission type } \\
\hline Non-elective & \multicolumn{4}{|c|}{ Reference } \\
\hline Elective & 1.65 & 1.52 & 1.79 & $<0.0001$ \\
\hline \multicolumn{5}{|l|}{ Admission day } \\
\hline Weekday & \multicolumn{4}{|c|}{ Reference } \\
\hline Weekend & 0.94 & 0.90 & 0.99 & 0.0134 \\
\hline \multicolumn{5}{|l|}{ Bed size of the hospital } \\
\hline Small & \multicolumn{4}{|c|}{ Reference } \\
\hline Medium & 1.61 & 1.49 & 1.74 & $<0.0001$ \\
\hline Large & 1.86 & 1.73 & 2.00 & $<0.0001$ \\
\hline \multicolumn{5}{|c|}{ Hospital Location \& Teaching Status } \\
\hline Rural & \multicolumn{4}{|c|}{ Reference } \\
\hline Urban Non-teaching & 2.67 & 2.43 & 2.94 & $<0.0001$ \\
\hline Urban Teaching & 2.98 & 2.71 & 3.28 & $<0.0001$ \\
\hline \multicolumn{5}{|l|}{ Hospital Region } \\
\hline Northeast & \multicolumn{4}{|c|}{ Reference } \\
\hline Midwest & 0.75 & 0.70 & 0.81 & $<0.0001$ \\
\hline South & 0.87 & 0.82 & 0.92 & $<0.0001$ \\
\hline West & 0.79 & 0.74 & 0.85 & $<0.0001$ \\
\hline \multicolumn{5}{|l|}{ Comorbidities of Patients } \\
\hline Hypertension & 0.93 & 0.89 & 0.98 & 0.0042 \\
\hline Diabetes & 0.88 & 0.83 & 0.93 & $<0.0001$ \\
\hline
\end{tabular}




\section{Cureus}

\begin{tabular}{|c|c|c|c|c|}
\hline Obesity & 0.81 & 0.76 & 0.87 & $<0.0001$ \\
\hline Hypercholesterolemia & 0.80 & 0.74 & 0.87 & $<0.0001$ \\
\hline Drug Abuse & 0.81 & 0.70 & 0.92 & 0.0016 \\
\hline Alcohol Abuse & 0.54 & 0.50 & 0.58 & $<0.0001$ \\
\hline Current or Past Smoker & 0.80 & 0.76 & 0.84 & $<0.0001$ \\
\hline Gallstone & 4.44 & 4.22 & 4.66 & $<0.0001$ \\
\hline Acquired ImmunoDeficiency Syndrome (AIDS) & 0.85 & 0.60 & 1.20 & 0.3567 \\
\hline End-Stage Renal Disease & 0.70 & 0.58 & 0.85 & 0.0004 \\
\hline Chronic Kidney Disease & 0.73 & 0.65 & 0.83 & $<0.0001$ \\
\hline Ischemic Heart Disease & 1.02 & 0.96 & 1.09 & 0.5800 \\
\hline Chronic Pancreatitis & 1.64 & 1.54 & 1.76 & $<0.0001$ \\
\hline Alcohol Withdrawal & 0.38 & 0.28 & 0.52 & $<0.0001$ \\
\hline \multicolumn{5}{|l|}{ Complications } \\
\hline Hypercalcemia & 0.72 & 0.51 & 1.02 & 0.0630 \\
\hline Acute Renal Failure & 0.92 & 0.84 & 1.01 & 0.0660 \\
\hline Shock & 1.02 & 0.80 & 1.30 & 0.8618 \\
\hline Systemic Inflammatory Response Syndrome & 1.26 & 1.11 & 1.44 & 0.0003 \\
\hline Ascites & 1.13 & 1.00 & 1.28 & 0.0507 \\
\hline Pleural Effusion & 1.87 & 1.23 & 2.85 & 0.0034 \\
\hline Respiratory Distress/Failure & 0.93 & 0.82 & 1.06 & 0.2899 \\
\hline Portal Vein Thrombosis & 1.65 & 1.22 & 2.22 & 0.0011 \\
\hline Deyo's Charlson Comorbidity Index (CCI) & 1.01 & 0.99 & 1.03 & 0.5537 \\
\hline Area Under the ROC Curve/C-Index & 0.77 & & & \\
\hline
\end{tabular}

TABLE 4: Predictors of endoscopic retrograde cholangiopancreatography (ERCP) utilization in acute pancreatitis hospitalizations

UL: upper limit; LL: lower limit

\section{Other outcomes}

Table 5 has mentioned the outcomes of ERCP utilization amongst acute pancreatitis patients. The patients with ERCP utilization in acute pancreatitis had a high prevalence of morbidity (8.81\% vs 3.34\%; $\mathrm{p}<0.0001)$, mortality $(1.10 \%$ vs $0.97 \%$; $p=0.0037)$, major/extreme disability ( $44.69 \%$ vs $29.43 \%$; $p<0.0001)$, discharge other than home ( $17.90 \%$ vs $12.82 \%$; $p<0.0001$ ), higher mean length of stay ( 8 days vs 5 days; LOSDiff $=+3$ days; $p<0.0001$ ), and cost of hospitalization $(\$ 56,337$ vs $\$ 31,260$; CostDiff $=+\$ 25,077 ; p<0.0001)$ than nonERCP utilization. 


\section{Cureus}

\begin{tabular}{|c|c|c|c|c|}
\hline & ERCP & Non-ERCP & Total & p-value \\
\hline Acute Pancreatitis weighted (\%) & $49107(1.87)$ & 2583202(98.13) & 2632309 (100) & $<0.0001$ \\
\hline Morbidity\# & $4325(8.81)$ & $86328(3.34)$ & $90653(3.44)$ & $<0.0001$ \\
\hline Mortality & $541(1.10)$ & $25107(0.97)$ & $25648(0.97)$ & 0.0037 \\
\hline Disability & & & & $<0.0001$ \\
\hline Minor/Moderate disability & $26692(55.31)$ & $1796651(70.57)$ & $1823343(70.29)$ & \\
\hline Major/Extreme disability & $21568(44.69)$ & $749259(29.43)$ & $770827(29.71)$ & \\
\hline Discharge Disposition & & & & $<0.0001$ \\
\hline Discharge to home & $39491(82.10)$ & 2154529 (87.18) & 2194020 (87.09) & \\
\hline Discharge other than home ${ }^{x}$ & $8608(17.90)$ & 316695 (12.82) & 325303 (12.91) & \\
\hline Length of Stay \pm SE (Days) & $8 \pm 0.10$ & $5 \pm 0.01$ & & $<0.0001$ \\
\hline Cost of Hospitalization \pm SE (\$) & $56337 \pm 866$ & $31260 \pm 76$ & & $<0.0001$ \\
\hline
\end{tabular}

\section{TABLE 5: Outcomes of endoscopic retrograde cholangiopancreatography (ERCP) utilization in acute pancreatitis hospitalizations}

* Discharge other than home: discharge to short term hospital, skilled nursing facility, or intermediate care facility, \# Morbidity defined as the length of stay >10days ( $>90$ percentile) and discharge other than home. The percentage in bracket is column $\%$ indicates a direct comparison between ERCP vs. Non-ERCP amongst acute pancreatitis patients.

\section{Discussion}

Our study analyzed the national inpatient trend, demographics, and outcomes of acute pancreatitis patients who utilized ERCP. Besides mortality and morbidity, we have evaluated the predictors of utilization and cost of hospitalization. To the best of our knowledge, this is the first large-scale study evaluating the trends and predictors of utilization of ERCP in this patient population. In our analysis, we observed a decreasing trend of ERCP utilization overall in patients with acute pancreatitis from 2003 to 2014. Mehta et al. have previously done a similar study, which showed a similar decreased overall trend of diagnostic utilization of ERCP, but the study specifically looked at utilization of ERCP in decompensated cirrhosis where it showed an increasing trend [6]. A recent population-based study by Somashekar et al. showed an increased prevalence of acute pancreatitis hospitalization (2002-2012). Another finding of this study was a declining frequency of acute pancreatitis with a gallstone-related disorder and increased acute pancreatitis hospitalization in association with chronic pancreatitis [7]. Our study did not look at the prevalence trend of gallstone-related acute pancreatitis; however, ERCP utilization was more associated with gallbladder etiology. The finding is interesting, as even though the prevalence of acute pancreatitis has increased, the ERCP trend has been consistently decreasing over the years. The reason for the decreasing trend of overall utilization for ERCP could be the increasing use of noninvasive diagnostic modalities of MRCP and endoscopic ultrasound [8].

Our result showed the increased utilization of ERCP in the female and old age population. Increasing age and female sex have been associated with a higher frequency of gallstone diseases, explaining the increased ERCP utilization. Increased ERCP use was also seen during acute pancreatitis hospitalization for whites, Hispanic, and Asian Pacific Islander race and is consistent with the results of the prior similar studies [9-10]. Another interesting finding of the increased ERCP utilization trend was also seen in larger bed size and urban teaching hospitals as compared to the rural and urban non-teaching hospitals. A retrospective population-based study done in 2014 to examine the disparities in colorectal cancer screening and treatment by comparing subspecialists distribution in rural and urban counties showed an increased density of gastroenterologists and other specialists in urban areas per 100,000 people as compared to rural areas [11-12]. Our study finding of comparatively less ERCP in small and rural hospitals likely reflects the resource-intense procedure nature of ERCP and the existing rural-urban disparity in the density of gastroenterologists. Similarly, inadequate subspecialist and related services coverage and availability, such as trained nursing staff and anesthesia services, could be the potential reason for the less utilization of ERCP trend on weekends compared to weekdays. The large urban and rural disparity concerns the availability of subspecialist services in rural areas, which also increases the cost burden on the health care system. [13-15] However, the results are based on limited data up till 2014. The increased number of new training programs in recent years will need further studies with recent years of data to assess real-time situations. 
A higher ERCP utilization association was observed in acute pancreatitis patients with ischemic heart disease and chronic pancreatitis. However, utilization in gallstone-related disease remains at the top. Previous studies have looked at the increased rate of association in acute pancreatitis and ischemic heart disease, suggesting ischemic heart disease-related chronic inflammatory state or statin and fibrates-related adverse events being the underlying mechanism [16-17]. This particular area to establish evidence of a strong association between the aforementioned conditions probably requires further studies. However, in our opinion, the finding itself is likely related to increased age group, as increased age is a commonly known risk factor for ischemic heart disease and showed increased utilization of ERCP likely because of higher case weight of acute pancreatitis in this age group. The patients who underwent ERCP procedures were also shown to have more complication-related codes registered during the hospitalization, especially SIRS, pleural effusion, and portal vein thrombosis. Higher odds of mortality and morbidity and LOS were significantly associated with patients who underwent ERCP as compared to non-ERCP patients. As the study showed a similar association with the ERCP group's complications, the data likely reflects the possibility of having more severe pancreatitis in these patients requiring further diagnostic and therapeutic evaluation with ERCP. A previous systematic survey of the prospective study suggested a higher incidence risk for morbidity and mortality with the ERCP procedure. However, in our study, the higher odds of complications in the ERCP utilization group do not establish any causal association because of the study design and make it difficult to ascertain whether these complications resulted from pancreatitis itself post procedure complications. In general, with the advancement in overall endoscopy techniques and training, ERCP has become a much safer and effective procedure overall. Many studies have proved that ERCP is a safe procedure, and a greater number of procedures are being done as an outpatient on the elective basis [12-15].

Overall, hospital utilization cost association, length of stay, and discharges other than home were higher in the ERCP utilization population than the non ERCP utilization population. Our study is one of the largest population-based cross-sectional studies to report the predictors of ERCP utilization and outcomes and complications profile of acute pancreatitis patients who underwent ERCP. Though NIS data is the largest national inpatient database with good statistical power, this study has limitations. This administrative database is obtained retrospectively by chart abstractions based on the discharge diagnosis codes, billing codes, etc. and hence is susceptible to coding errors. The diagnosis of acute pancreatitis and other complications in the NIS database are physicians documented with clinical evidence. Complications and outcomes depend on the severity of the disease, and lab values are crucial to deciding severity and are missing. We have not followed up on the patient's post-hospitalization to evaluate the disability. Due to the study's nature, we cannot establish the temporal relationship between procedure and complications, so we can only comment on complications amongst acute pancreatitis patients who utilized ERCP.

\section{Conclusions}

This study showed a decreasing overall trend of ERCP but a higher length of stay, cost of utilization in hospital admission, and discrepancy between urban and rural utilization. One way to decrease utilization cost along with the length of stay is to avoid unnecessary procedures in cases where suspicion for biliary etiology is low and using less invasive and cheaper alternatives compared to ERCP. More studies related to decision analysis are required to evaluate the importance of specific use of ERCP and other hepatobiliary procedures in order to mitigate the healthcare cost burden.

\section{Additional Information \\ Disclosures}

Human subjects: All authors have confirmed that this study did not involve human participants or tissue. Animal subjects: All authors have confirmed that this study did not involve animal subjects or tissue. Conflicts of interest: In compliance with the ICMJE uniform disclosure form, all authors declare the following: Payment/services info: All authors have declared that no financial support was received from any organization for the submitted work. Financial relationships: All authors have declared that they have no financial relationships at present or within the previous three years with any organizations that might have an interest in the submitted work. Other relationships: All authors have declared that there are no other relationships or activities that could appear to have influenced the submitted work.

\section{References}

1. Peery AF, Crockett SD, Murphy CC, et al.: Burden and cost of gastrointestinal, liver, and pancreatic diseases in the United States: update 2018. Gastroenterology. 2019, 156:254-272. 10.1053/j.gastro.2018.08.063

2. Jamal MM, Yoon EJ, Saadi A, Sy TY, Hashemzadeh M: Trends in the utilization of endoscopic retrograde cholangiopancreatography (ERCP) in the United States. Am J Gastroenterol. 2007, 102:966-975.

3. Munigala S, Yadav D: Case-fatality from acute pancreatitis is decreasing, but its population mortality shows little change. Pancreatology. 2016, 16:542-550. 10.1016/j.pan.2016.04.008

4. Malli A, Durkin C, Groce JR, Hinton A, Conwell DL, Krishna SG: Unavailability of endoscopic retrograde cholangiography adversely impacts hospital outcomes of acute biliary pancreatitis. A national survey and propensity-matched analysis. Pancreas. 2020, 49:39-45. 10.1097/MPA.0000000000001435

5. Patel U, Kodumuri N, Malik P, et al.: Hypocalcemia and vitamin D deficiency amongst migraine patients: a nationwide retrospective study. Medicina. 2019, 55:407-410. 10.3390/medicina55080407

6. Mehta D, Poojary P, Saha A, et al.: National trends of endoscopic retrograde cholangiopancreatography 
utilization and outcomes in decompensated cirrhosis. Surg Endosc. 2019, 33:169-178. 10.1007/s00464-0186290-1

7. Krishna SG, Kamboj AK, Hart PA, Hinton A, Conwell DL: The changing epidemiology of acute pancreatitis hospitalizations: a decade of trends and the impact of chronic pancreatitis. Pancreas. 2017, 46:482-488. 10.1097/MPA.0000000000000783

8. Moffatt DC, Yu BN, Yie W, Bernstein CN: Trends in utilization of diagnostic and therapeutic ERCP and cholecystectomy over the past 25 years: a population-based study. Gastrointest Endosc. 2014, 79:615-622. 10.1016/j.gie.2013.08.028

9. Maurer KR, Everhart JE, Ezzati TM, et al.: Prevalence of gallstone disease in Hispanic populations in the United States. Gastroenterology. 1989, 96:487-492. 10.1016/0016-5085(89)91575-8

10. Sichieri R, Everhart JE, Roth HP: Low incidence of hospitalization with gallbladder disease among blacks in the United States. Am J Epidemiol. 1990, 131:826-835. 10.1093/oxfordjournals.aje.a115573

11. Aboagye JK, Kaiser HE, Hayanga AJ: Rural-urban differences in access to specialist providers of colorectal cancer care in the United States. A physician workforce issue. JAMA Surg. 2014, 149:537-543. 10.1001/jamasurg.2013.5062

12. Jeurnink SM, Poley JW, Steyerberg EW, Kuipers EJ, Siersema PD: ERCP as an outpatient treatment: a review . Gastrointest Endosc. 2008, 68:118-123. 10.1016/j.gie.2007.11.035

13. Freeman ML, Nelson DB, Sherman S, et al.: Same-day discharge after endoscopic biliary sphincterotomy: observations from a prospective multicenter complication study. Gastrointest Endosc. 1999, 49:580-586. 10.1016/S0016-5107(99)70385-8

14. Duncan HD, Hodgkinson L, Deakin M, Green JRB: The safety of diagnostic and therapeutic ERCP as a daycase procedure with a selective admission policy. Eur J Gastroenterol Hepatol. 1997, 9:905-908. 10.1097/00042737-199709000-00015

15. Andriulli A, Loperfido S, Napolitano G, et al.: Incidence rates of post-ERCP complications: A systematic survey of prospective studies. Am J Gastroenterol. 2007, 102:1781-1788.

16. Copeland LA, Scott Swendsen C, Sears DM, MacCarthy AA, McNeal CJ: Association between triglyceride levels and cardiovascular disease in patients with acute pancreatitis. PLoS One. 2018, 13:e0179998. 10.1371/journal.pone.0179998

17. Bexelius TS, Ljung R, Mattsson F, Lagergren J: Cardiovascular disease and risk of acute pancreatitis in a population-based study. Pancreas. 2013, 42:1011-1015. 10.1097/MPA.0b013e318287cb62 